\title{
TRPM8 voltage sensor mutants reveal a mechanism for integrating thermal and chemical stimuli
}

\author{
Thomas Voets, Grzegorz Owsianik, Annelies Janssens, Karel Talavera \& Bernd Nilius
}

TRPM8, a member of the transient receptor potential (TRP) channel superfamily, is expressed in thermosensitive neurons, in which it functions as a cold and menthol sensor. TRPM8 and most other temperature-sensitive TRP channels (thermoTRPs) are voltage gated; temperature and ligands regulate channel opening by shifting the voltage dependence of activation. The mechanisms and structures underlying gating of thermoTRPs are currently poorly understood. Here we show that charge-neutralizing mutations in transmembrane segment 4 (S4) and the S4-S5 linker of human TRPM8 reduce the channel's gating charge, which indicates that this region is part of the voltage sensor. Mutagenesis-induced changes in voltage sensitivity translated into altered thermal sensitivity, thereby establishing the strict coupling between voltage and temperature sensing. Specific mutations in this region also affected menthol affinity, which indicates a direct interaction between menthol and the TRPM8 voltage sensor. Based on these findings, we present a Monod-Wyman-Changeux-type model explaining the combined effects of voltage, temperature and menthol on TRPM8 gating.

Mammals detect changes in environmental temperature from noxious cold $\left(<8{ }^{\circ} \mathrm{C}\right)$ to noxious heat $\left(>52{ }^{\circ} \mathrm{C}\right)$ through sensory nerve endings in the skin and mouth ${ }^{1}$ and through skin keratinocytes ${ }^{2}$. Recently, thermoTRPs have been put forward as the main temperature sensors in thermosensory cells ${ }^{3-5}$. Eight different thermoTRPs have been characterized in mammals, with thermal sensitivities that cover the temperature range between noxious cold and noxious heat ${ }^{4-7}$. The mechanisms and structures that underlie the steep temperature dependence of these TRP channels are poorly understood.

We have recently shown that the heat-activated TRPV1 and the cold-activated TRPM8 are voltage-gated channels activated upon membrane depolarization, and that thermal activation reflects a robust but graded shift of the voltage dependence of activation from strongly depolarized potentials toward the physiological potential range $^{8}$. Similar observations have been made for other thermoTRPs ${ }^{7,9}$. Moreover, a growing list of chemical agonists of different thermoTRPs, including menthol (1; TRPM8), capsaicin (2; TRPV1), camphor (3; TRPV1 and TRPV3) and phosphatidylinositol-4,5-bisphosphate (TRPM8 and TRPM4), have been shown to induce channel activation by shifting the voltage dependence of activation ${ }^{2,8-13}$. Thus, explaining how thermoTRPs detect chemical and thermal stimuli requires an understanding of the mechanisms and structures underlying voltage sensing.

In seeking to identify residues involved in gating of TRPM8 (refs. 14,15), we were guided by the structural similarities between TRP channels and voltage-gated potassium $(\mathrm{Kv})$ channels. Both classes of cation channels are tetramers of four identical or similar domains, with six transmembrane segments (S1-S6) and cytosolic $\mathrm{N}$ - and C-terminal tails ${ }^{16,17}$. In Kv channels, the voltage sensor consists of a cluster of positively charged residues located in S4 (refs. 18-20). Movement of this sensor relative to the transmembrane electrical field results in the gating of the channel pore, which consists of S5, S6 and the interconnecting P-loop ${ }^{18,21}$. Recent structural data suggest that the S4-S5 linker interacts with the C-terminal part of S6, such that movement of this linker leads to constriction or dilatation of the intracellular entrance of the channel pore ${ }^{22}$. In this report, we show that S4 and the S4-S5 linker of TRPM8 have a role in voltage sensing, and we provide evidence that menthol acts on the channel through a direct interaction with the voltage sensor region.

\section{RESULTS}

Mutations in S4 and S4-S5 linker alter voltage dependence Sequence alignment of TRPM8 with Kv1.2, a Shaker-type channel whose crystal structure has recently been elucidated ${ }^{22,23}$, revealed the highest degree of homology ( $31 \%$ identity, 52\% similarity) in a region corresponding to S4 and the S4-S5 linker (Fig. 1a,b). Notably, we found that only one of the four positively charged arginines that contribute to gating charge in Shaker-type $\mathrm{K}^{+}$channels is conserved in TRPM8 (Arg842; Fig. 1a,b). To investigate the role of this region in the gating of TRPM8, we changed all positively charged residues (Fig. 1a) to the neutral residue alanine and tested the effect of the mutations on the sensitivity of TRPM8 to voltage, temperature and menthol. We also mutated a single histidine, as histidine can be positively charged depending on its chemical environment. All chargeneutralizing mutants formed functional channels upon expression in human embryonic kidney (HEK) 293 cells (Fig. 1c), with the exception of R851A, for which we were not able to measure currents above background (Supplementary Table 1 online). Introducing a 
a

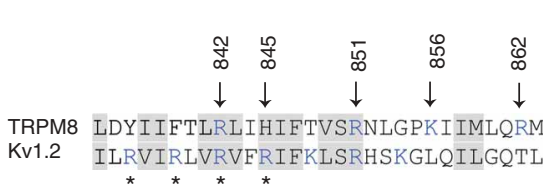

b

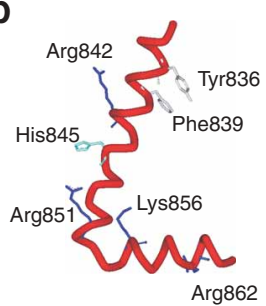

Figure 1 Charge neutralizations in S4 and the S4-S5 linker of TRPM8. (a) Sequence alignment of TRPM8 and Kv1.2. Gray background marks sequence conservation, blue lettering indicates positive charges, arrows indicate the residues mutated in this study and asterisks indicate charges that contribute to voltage sensing in Kv channels. (b) Tentative structural model of S4 and the S4-S5 linker of TRPM8 based on the Kv1.2 coordinates. Kv1.2 contains two voltage-sensing arginines at the positions corresponding to Tyr836 and Phe839 in TRPM8. (c) Wholecell current traces at $25{ }^{\circ} \mathrm{C}$ in response to the indicated voltage protocol. A P/8 subtraction protocol around a holding potential of $-160 \mathrm{mV}$ was used to subtract the linear capacitive and leak currents. (d) Steady state activation curves. (e,f) $V_{1 / 2}$ and $z_{\text {app }}$ at $25^{\circ} \mathrm{C}$. Mutant R862A (gray bar) did not reach half-maximal activation at potentials up to $+260 \mathrm{mV}$, making an estimation of $V_{1 / 2}$ and $z_{\text {app }}$ unfeasible. Error bars indicate s.e.m. $\left(n>5\right.$; $\left.{ }^{* *} P<0.01 ;{ }^{* *} P<0.001\right)$. WT, wild type; GFP, green fluorescent protein.

glutamine at this position (R851Q) did, however, yield a functional channel, which we used for further evaluation (Fig. 1c). In addition, we produced the corresponding charge-conserving mutants, all of which expressed as functional channels (Supplementary Table 1).

We analyzed the effects of the mutations on the voltage dependence of TRPM8 activation at $25{ }^{\circ} \mathrm{C}$ using voltage-step protocols covering the voltage range -120 to $+260 \mathrm{mV}$ (Fig. 1c). The apparent channel open probability $\left(P_{\text {open }}\right)$ was determined as $G / G_{\max }$, where $G$ represents the steady state conductance and $G_{\max }$ represents the saturating conductance measured at strongly depolarized potentials in the presence of $1 \mathrm{mM}$ menthol (for more details, see Methods and Supplementary Fig. 1 online). In our initial analysis, we assumed a two-state channel-gating model with one open state and one closed state $^{8}$ (for a detailed comparison with an alternative eightstate gating model ${ }^{24}$, see Supplementary Methods and Supplementary Fig. 2 online). Accordingly, we fitted activation curves using a Boltzmann function:

$$
P_{\text {open }}=\frac{1}{1+\exp \left(-\frac{z}{k_{\mathrm{B}} T}\left(V-V_{1 / 2}\right)\right)}
$$

where $z$ is the gating charge (in elementary charge units: $e_{\mathrm{o}}=1.6 \times$ $\left.10^{-19} \mathrm{C}\right), V$ is voltage, $V_{1 / 2}$ is the voltage for half-maximal activation, $k_{\mathrm{B}}$ is the Boltzmann constant $\left(1.38 \times 10^{-23} \mathrm{~J} \mathrm{~K}^{-1}\right)$ and $T$ is the absolute temperature (Fig. 1d-f). $V_{1 / 2}$ is further determined by

$$
V_{1 / 2}=\Delta G /(z F)=(\Delta H-T \Delta S) /(z F)
$$

where $F$ represents the Faraday constant $\left(9.65 \times 10^{-4} \mathrm{C} \mathrm{mol}^{-1}\right)$ and $\Delta G$ represents the difference in free energy between the open and closed states, which consists of an enthalpic $(\Delta H)$ and an entropic $(-T \Delta S)$ component ${ }^{6,25}$. We used $z_{\text {app }}$ (apparent valence) to refer to the gating charge estimates determined using equation (1), because the accuracy of this estimate depends on the validity of the two-state model.

Two charge-neutralizing mutants (R842A and K856A) had a $z_{\text {app }}$ value that was significantly lower than that of the wild type (Fig. 1f), which in case of strict applicability of the two-state model indicates a smaller gating charge. Moreover, all charge-neutralizing mutations
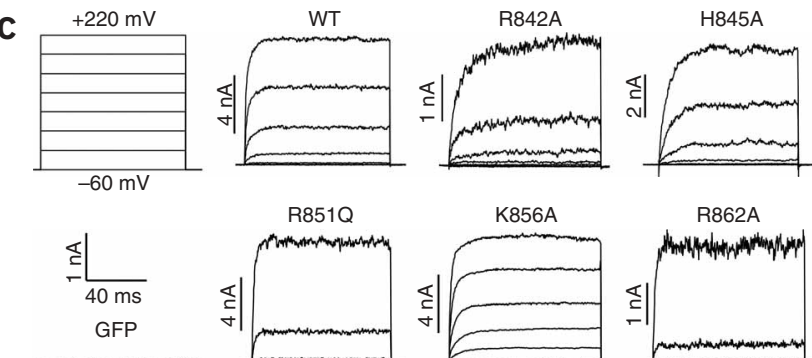

$\mathrm{R} 851 \mathrm{Q}$
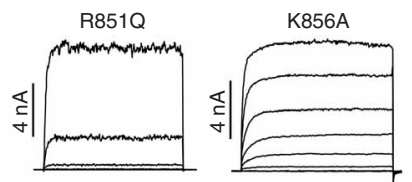

R862A
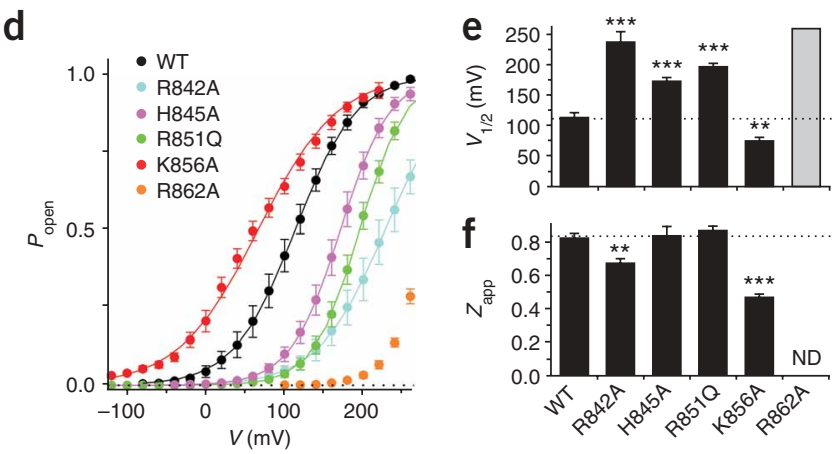

and one charge-conserving mutation substantially affected the position of the voltage dependence of channel activation: $V_{1 / 2}$ was significantly shifted toward more positive potentials for R842A, H845A, R851Q, R862A and K856R, and was shifted toward more negative voltages for K856A (Fig. 1d,e). These shifts indicate substantial changes in the relative stability of the closed and open states, as quantified by the changes in $\Delta G$ (Supplementary Table 1 ).

\section{Arg842 and Lys856 contribute to gating charge}

To obtain a more rigorous estimate of gating charge we used the limiting slope method (LSM), whose results are model-independent $^{18,26,27}$. LSM entails calculating the activation charge displacement, $q_{\mathrm{a}}(V)$, from the slope of $\ln \left(P_{\text {open }}\right)$ versus $V$, according to:

$$
q_{\mathrm{a}}(V)=k_{\mathrm{B}} T \frac{\mathrm{d} \ln \left(P_{\mathrm{open}}(V)\right)}{\mathrm{d} V}
$$

Then, the total gating charge $\left(z_{\mathrm{T}}\right)$ is determined as the asymptotic value of $q_{\mathrm{a}}(V)$ at low $P_{\text {open. }}$. Thus, we applied slow voltage ramps covering the voltage range for which $P_{\text {open }}$ is expected to be low (approximately between $10^{-4}$ and $10^{-1}$ ). Leakage currents at very negative potentials $(-250$ to $-220 \mathrm{mV}$ ) were fitted with a linear function and subtracted from the current record to obtain the TRPM8 current (Fig. 2a) ${ }^{20}$. Dividing the ionic current by the applied voltage yields the ionic conductance ( $G$; Fig. $2 \mathbf{2 b}$ ), which is divided by $G_{\max }$ to obtain the apparent $P_{\text {open }}$ (Fig. 2c). It should be noted that $\mathrm{d} \ln \left(P_{\text {open }}\right) / \mathrm{d} V$ is independent of $G_{\text {max }}$, and hence determination of $z_{\mathrm{T}}$ is still accurate in the event that $G_{\max }$ is underestimated. For wild-type TRPM8, $q_{\mathrm{a}}(V)$ saturates at a $z_{\mathrm{T}}$ of $0.89 \pm 0.03 e_{\mathrm{o}}(n=8$; error is s.e.m. $)$ for $P_{\text {open }}$ values below $\sim 10^{-1}$ (Fig. 2d,e). For the K856A mutant, $q_{\mathrm{a}}(V)$ also saturates, but at a significantly lower $z_{\mathrm{T}}$ value of $0.62 \pm 0.03$ $e_{\mathrm{o}}(n=7 ; P<0.01)$ (Fig. 2c-e). A similar reduction in $z_{\mathrm{T}}$ was observed for the R842A mutant, whereas all other charge-neutralizing mutations in the tested region had no noticeable effect on $z_{\mathrm{T}}$ (Fig. 2e). We also analyzed charge-conserving mutations at these positions (R842K and K856R) and found that they do not reduce the total gating charge (Fig. 2e and Supplementary Table 1). Taken together, 

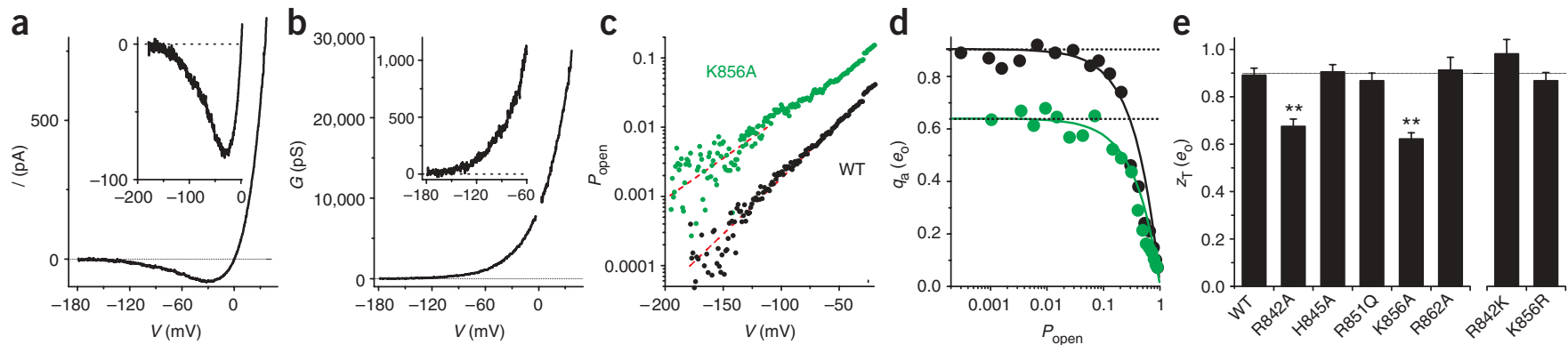

Figure 2 Determination of the total gating charge with the limited slope method. (a) Leak-subtracted whole-cell TRPM8 current measured during 2-s voltage ramps from +60 to $-220 \mathrm{mV}$. Five consecutive current traces were averaged to improve the signal-to-noise ratio. (b) Whole-cell conductance for the recording in $\mathbf{a}$. The insets in $\mathbf{a}$ and $\mathbf{b}$ show an expanded view on the current and conductance in the lower voltage range. (c) Examples of $P_{\text {open }}$ as a function of voltage for wild-type TRPM8 and K856A. Red dashed lines represent exponential fits for $P_{\text {open }}<10^{-2}$. (d) $q_{\mathrm{a}}$ plotted as a function of $P_{\text {open }}$ for the data in $\mathbf{c}$. Data points represent the slope of $\ln \left(P_{\text {open }}\right)$ versus $V$ determined for overlapping 20-mV segments. Solid lines represent the expected relationship for the two-state model. (e) Total gating charge $\left(z_{T}\right)$ for wild-type TRPM8 and the indicated mutants. Error bars indicate s.e.m. $\left(n>5 ;{ }^{* *} P<0.01\right)$.

these data demonstrate that neutralization of positive charges at positions 842 and 856 reduces the gating charge movement of TRPM8. When the effects of mutations R842A and K856A on $z_{\text {app }}$ or $z_{\mathrm{T}}$ are directly added up, it follows that these residues may account for the majority of the gating charge $(\sim 50-70 \%)$, but not all of it. We also tested the double mutant R842A K856A, but this protein was no longer functional (Supplementary Table 1).

Note that the gating charge estimates obtained from the Boltzmann fits $\left(z_{\text {app }}\right.$; Fig. 1f) closely approach the $z_{\mathrm{T}}$ values obtained with the LSM method (Fig. 2e and Supplementary Table 1). Moreover, the relation between $q_{\mathrm{a}}$ and $P_{\text {open }}$ was reasonably well predicted by the two-state model (Fig. 2d). These properties further validate the use of this simple model to approximate the temperature-dependent gating behavior of TRPM8 (ref. 8). In all further calculations we used the $z_{\mathrm{T}}$ values for gating charge, because $z_{\mathrm{T}}$ is model-independent and is determined at low $P_{\text {open }}$, which effectively reduces the influence of potential voltage-clamp errors.
Mutations in S4 and S4-S5 linker alter temperature sensitivity

We next tested the effect of the charge-neutralizing mutations on activation of TRPM8 by cooling. First, we performed intracellular $\mathrm{Ca}^{2+}\left(\left[\mathrm{Ca}^{2+}\right]_{\mathrm{i}}\right)$ measurements on HEK 293 cells transfected with wildtype or mutant TRPM8 while cooling the bath from $40{ }^{\circ} \mathrm{C}$ to $10{ }^{\circ} \mathrm{C}$. This approach allows detection of changes in thermal sensitivity in intact cells and determination of an apparent thermal threshold, which is defined as the temperature at which the increase in $\left[\mathrm{Ca}^{2+}\right]_{\mathrm{i}}$ is significantly higher than that in nontransfected cells. In line with previous studies ${ }^{14,15}$, cells transfected with wild-type TRPM8 responded to cooling with a robust increase in $\left[\mathrm{Ca}^{2+}\right]_{i}$ and an apparent thermal threshold of $28 \pm 3{ }^{\circ} \mathrm{C}(n=7$; Fig. 3a-c). The charge-neutralizing mutations strongly affected the temperature sensitivity of TRPM8 (Fig. 3a,b). The threshold for cold activation of mutant K856A was shifted to warmer temperatures $\left(35 \pm 2{ }^{\circ} \mathrm{C} ; n=8\right.$; $P<0.01)$, whereas all other mutants activated at significantly $(P<0.01)$ lower temperatures relative to wild-type TRPM8 a

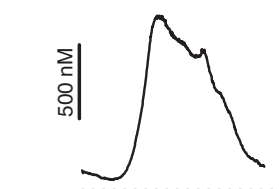

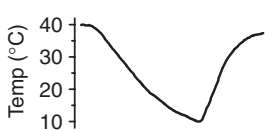

$\mathrm{R} 851 \mathrm{Q}$

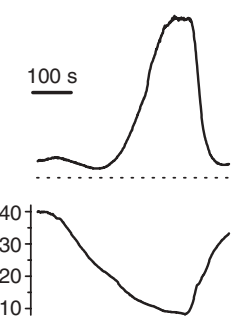

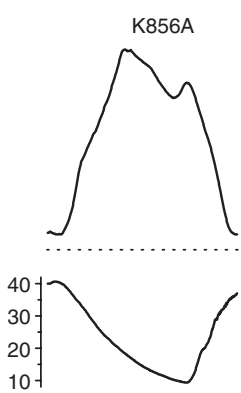

$10-$

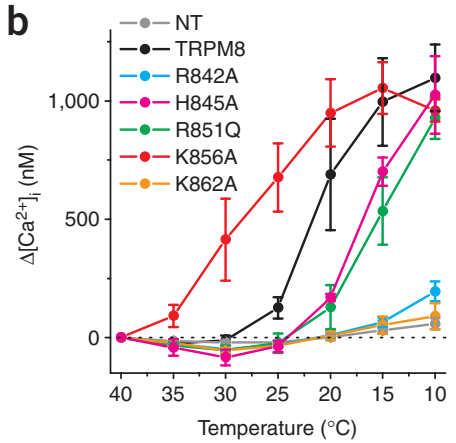

d

Figure 3 Charge neutralizations in S4 and the S4-S5 linker alter the temperature dependence of TRPM8. (a) $\left[\mathrm{Ca}^{2+}\right]_{i}$ measurements during cooling for wild-type TRPM8 and the indicated mutants. (b) Average increase in $\left[\mathrm{Ca}^{2+}\right]_{i}$ for nontransfected (NT) cells and cells transfected with wild-type TRPM8 and the indicated mutants. (c) Correlation between the apparent thermal threshold and $V_{1 / 2}$ at $25{ }^{\circ} \mathrm{C}$. The apparent thermal threshold was defined as the temperature at which the increase in $\left[\mathrm{Ca}^{2+}\right]_{\mathrm{i}}$ is significantly higher than that in nontransfected cells. (d) $V_{1 / 2}$ as a function of temperature for wild-type TRPM 8 and the indicated mutants. Same color code as in b. (e) Outward currents at different temperatures for Arg842 and Arg862. Error bars indicate s.e.m. $(n>5)$.

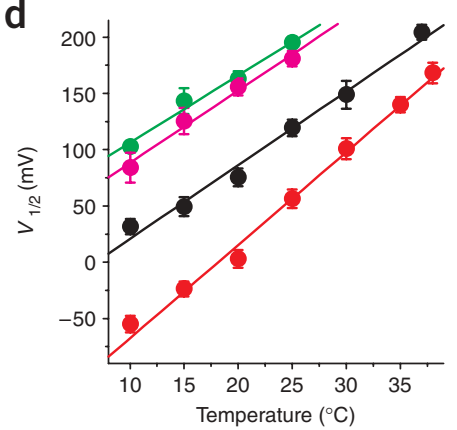

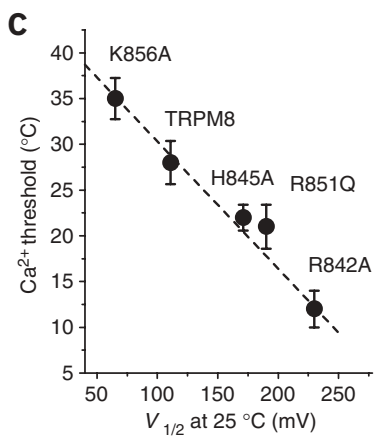

e

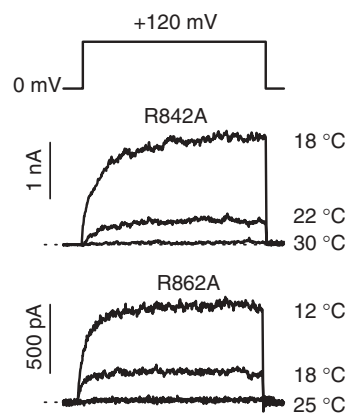


a

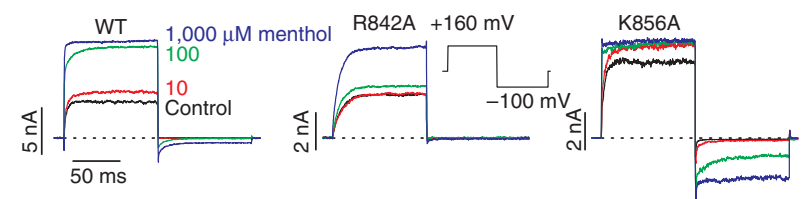

C

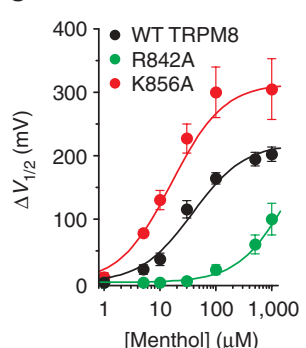

d

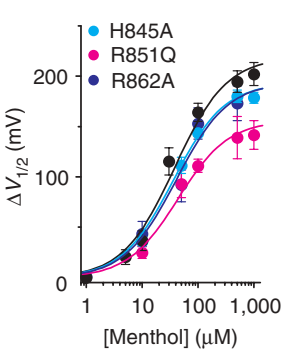

e

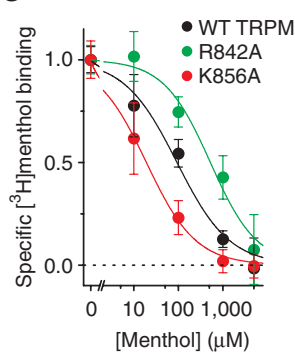

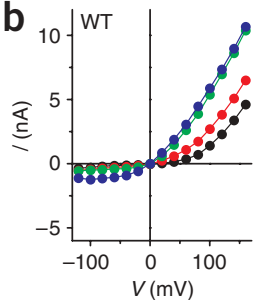
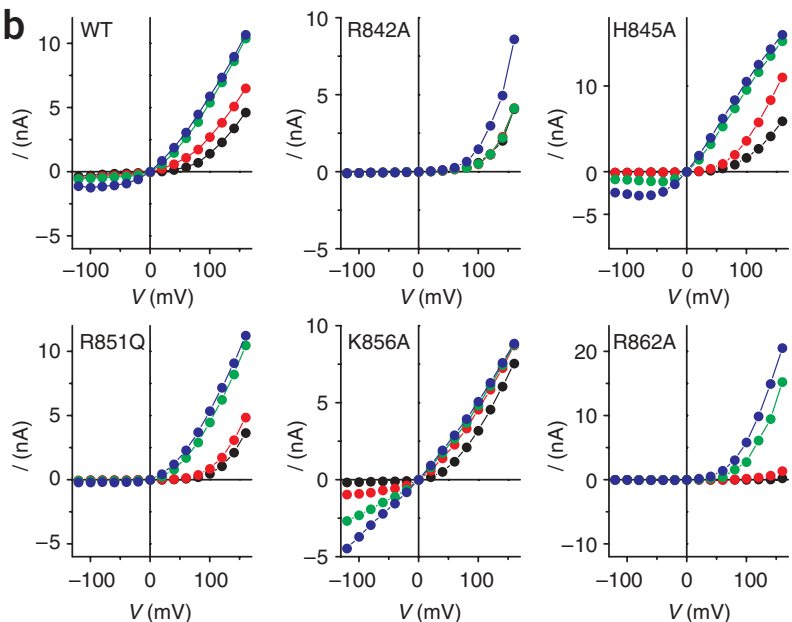

Figure 4 Residues in S4 and the S4-S5 linker determine the menthol sensitivity of TRPM8. (a) Current traces in response to the indicated voltage protocol for wild-type and mutant TRPM8 at $25^{\circ} \mathrm{C}$, in the presence of $0,10,100$ and 1,000 $\mu \mathrm{M}$ menthol. (b) Steady state current-voltage relations for wild-type and mutant TRPM8 with 0, 10, 100 and 1,000 $\mu \mathrm{M}$ menthol. Same color code as in $\mathbf{a}$. (c,d) Leftward shift of $V_{1 / 2}\left(\Delta V_{1 / 2}\right)$ as a function of menthol concentration for wild-type, R842A and K856A TRPM8 (c) and for H845A, R851Q and R862A TRPM8 (d). Solid lines represent the best fit to the data using the MWC model. (e) Inhibition of specific $\left[{ }^{3} \mathrm{H}\right]$ menthol binding to membranes containing wild-type or mutant TRPM8 by nonradioactive menthol. Error bars indicate s.e.m. $(n>5)$.

(Fig. 3a,b). As predicted by the two-state model, changes in apparent thermal thresholds correlated well with the changes in $V_{1 / 2}$ (Fig. 3c).

We have previously shown that changes in temperature activate TRPM8 and other thermoTRPs (TRPV1, TRPM4 and TRPM5) ${ }^{7,8}$ by causing a substantial shift of the voltage dependence of activation. Between $10{ }^{\circ} \mathrm{C}$ and $37{ }^{\circ} \mathrm{C}$ we found a linear relation between $V_{1 / 2}$ and $T$ for wild-type TRPM8 and several mutants, which suggests that $\Delta H$ and $\Delta S$ are relatively constant in the tested temperature range (Fig. $3 \mathbf{d}$ ). From equation (2), it then follows that $\mathrm{d} V_{1 / 2} / \mathrm{d} T=-\Delta S /(z F)$. Thus, the increase in $V_{1 / 2}$ upon heating implies that opening of TRPM8 leads to a reduction in entropy. For wild-type TRPM8, $\mathrm{d} V_{1 / 2} / \mathrm{d} T$ amounted to $6.5 \pm 0.3 \mathrm{mV}^{\circ} \mathrm{C}^{-1}$. Taking into account a $z_{\mathrm{T}}$ of 0.89 equivalent charges, this corresponds to a $\Delta S$ of $-560 \pm 40 \mathrm{~J} \mathrm{~mol}^{-1} \mathrm{~K}^{-1}(n=8)$. Similar values were obtained for mutants of non-voltage-sensing residues H845A and R851Q (Fig. 3d and Supplementary Table 1). Importantly, mutant K856A showed a temperature dependence that was significantly steeper than that of the wild type $\left(\mathrm{d} V_{1 / 2} / \mathrm{d} T=8.4 \pm 0.5 \mathrm{mV}{ }^{\circ} \mathrm{C}^{-1} ; n=\right.$ 9; $P<0.01$; Fig. 3d), an observation that is accounted for by the lower $z_{\mathrm{T}}$ of this mutant, as $\Delta S$ was not significantly different from that of the wild type $\left(-520 \pm 50 \mathrm{~J} \mathrm{~mol}^{-1} \mathrm{~K}^{-1}\right)$. In the case of charge-neutralizing mutants R842A and R862A and the charge-conserving mutant K856R, the voltage dependence of activation was shifted to such extremely positive voltages that determination of $V_{1 / 2}$ in function of temperature was unworkable. Nevertheless, these mutants showed cold-induced current activation at depolarized potentials (Fig. 3e). For the other charge-conserving mutants- $\mathrm{R} 842 \mathrm{~K}, \mathrm{R} 851 \mathrm{~K}$ and $\mathrm{R} 862 \mathrm{~K}$ - values for $\mathrm{d} V_{1 / 2} / \mathrm{d} T$ and $\Delta S$ were not significantly different from those of the wild type (Supplementary Table 1 ).

\section{Mutations in S4 and the S4-S5 linker affect menthol sensitivity}

Next, we investigated the effect of mutations in S4 and the S4-S5 linker on the sensitivity of TRPM8 to the cooling compound menthol. Menthol shifts the TRPM8 activation curve toward physiological voltages, thereby mimicking the effect of $\operatorname{cold}^{8}$. The effect of menthol on $V_{1 / 2}$ is dose-dependent ${ }^{8}$; fitting a Hill function to the data yielded a concentration for half-maximal effect $\left(\mathrm{EC}_{50}\right)$ of $29 \pm 3 \mu \mathrm{M}$ and a maximal shift of $208 \pm 8 \mathrm{mV}(n=8$; Fig. 4a-c). Similar menthol sensitivity was found for H845A, R851Q and R862A (Fig. 4b,d and Supplementary Table 1). However, mutant R842A showed no response to concentrations below $100 \mu \mathrm{M}$ (Fig. 4a,b), and the $\mathrm{EC}_{50}$ for this mutant amounted to $820 \pm 220 \mu \mathrm{M}\left(n=8 ; P<10^{-5}\right.$; Fig. 4c). Likewise, other substitutions at this position (mutants $\mathrm{R} 842 \mathrm{~K}$ and $\mathrm{R} 842 \mathrm{H})$ led to significantly higher $\mathrm{EC}_{50}$ values $(P<0.01$ and $P<10^{-5}$, respectively; Supplementary Table 1). In contrast, K856A showed a higher sensitivity to menthol (Fig. $4 \mathbf{a}-\mathbf{c}$ ), with an $\mathrm{EC}_{50}$ of $11.7 \pm 2.6 \mu \mathrm{M}(n=5 ; P<0.01)$. Moreover, the maximal shift induced by menthol for K856A was significantly higher than for wildtype TRPM8 $(320 \pm 40 \mathrm{mV} ; n=8 ; P<0.01)$. The $\mathrm{EC}_{50}$ value obtained for the charge-conserving mutant K856R was not significantly different from that of the wild type (Supplementary Table 1).

\section{Mutations in S4 and S4-S5 linker affect $\left[{ }^{3} \mathrm{H}\right]$ menthol binding}

How does menthol interact with TRPM8? Menthol directly and rapidly activates TRPM8 in cell-free inside-out patches ${ }^{8}$, thereby demonstrating a membrane-delimited mode of action. These observations are consistent with direct binding of menthol to the channel, but they do not fully exclude the possibility that menthol acts via an unknown membrane-associated protein or even through a nonspecific effect of menthol on the membrane phospholipids. To investigate these possibilities we performed radioligand binding and displacement studies using $\left[{ }^{3} \mathrm{H}\right]$ menthol on plasma membrane-enriched fractions from HEK 293 cells expressing wild-type and mutant TRPM8 (ref. 28). These membranes fractions were incubated with $\left[{ }^{3} \mathrm{H}\right]$ menthol in the presence of different concentrations of cold (nonlabeled) menthol. No specific $\left[{ }^{3} \mathrm{H}\right]$ menthol displacement was observed in nontransfected cells or in cells expressing TRPM2, the closest homolog of TRPM8 (Supplementary Fig. 3 online). In contrast, plasma membrane-enriched fractions from cells expressing wild-type TRPM8 showed substantial specific $\left[{ }^{3} \mathrm{H}\right]$ menthol binding (Supplementary Fig. 3), an effect that was inhibited by nonlabeled menthol with a half-maximal inhibitory concentration $\left(\mathrm{IC}_{50}\right)$ of $92 \pm 25 \mu \mathrm{M}$ $\left(n=9\right.$; Fig. 4e). In the case of mutant K856A, we obtained a lower $\mathrm{IC}_{50}$ 
a

TRPM5 TVLAIDFMVFTLRLIHIFAIHKQLGPKI I IVERMMK TRPM8 VIFCLDYIIFTLRLIHIFTVSRNLGPKIIMLQRMLI
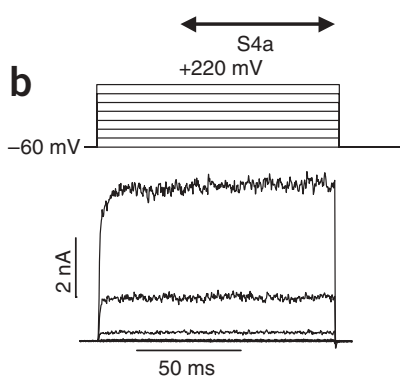

d

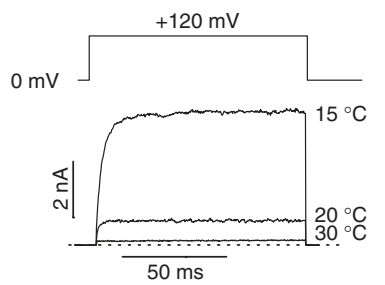

f
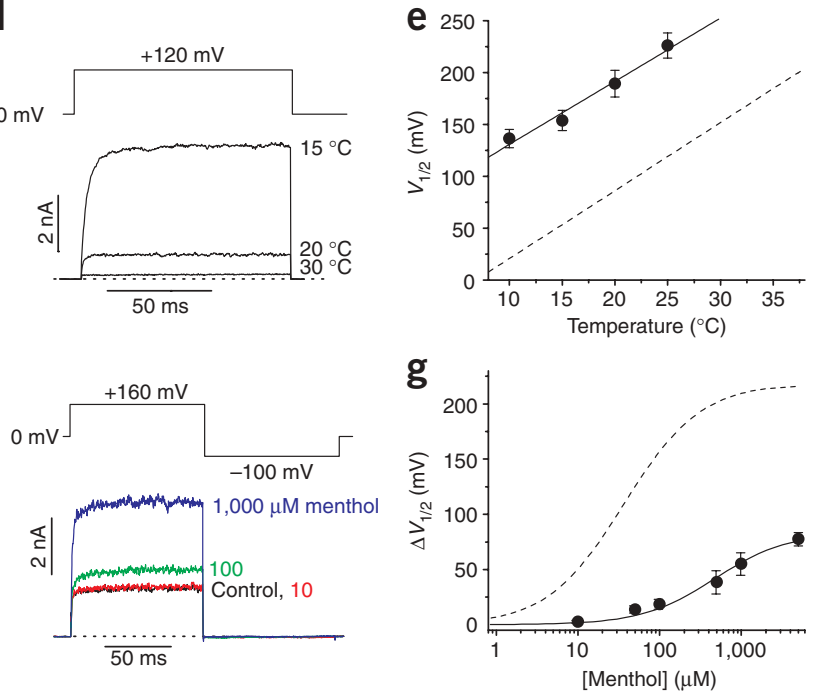

Figure 5 TRPM8-TRPM5 chimeras. (a) Sequence alignment of S4 and the S4-S5 linker of TRPM5 and TRPM8. Yellow background marks sequence identity, gray background marks sequence conservation and blue lettering indicates positive charges. The arrows indicate segments that were transferred from TRPM5 to TRPM8 to produce the S4a_M5, S4b_M5 and S4c_M5 mutants. (b) Whole-cell current traces at $25^{\circ} \mathrm{C}$ in response to the indicated voltage protocol for S4b_M5. (c) Steady state activation curves at $25{ }^{\circ} \mathrm{C}$ for S4b_M5. (d) Outward currents at different temperatures for S4b_M5. (e) $V_{1 / 2}$ as a function of temperature for S4b_M5. (f) Current traces in response to the indicated voltage protocol for S4b_M5 at $25{ }^{\circ} \mathrm{C}$, in the presence of $0,10,100$ and $1,000 \mu \mathrm{M}$ menthol. (g) Leftward shift of $V_{1 / 2}\left(\Delta V_{1 / 2}\right)$ as a function of menthol concentration for S4b_M5. The solid line represents the best fit to the data using the MWC model. The dotted lines in $\mathbf{c}$, e and $\mathbf{g}$ represent the corresponding average values for wild-type TRPM8. Error bars indicate s.e.m. $(n>5)$.

of $19.0 \pm 1.7 \mu \mathrm{M}(n=12 ; P<0.01)$, whereas for mutant R842A the $\mathrm{IC}_{50}$ was increased to $520 \pm 120 \mu \mathrm{M}(n=12 ; P<0.001$; Fig. 4e $)$.

We also tested $\left[{ }^{3} \mathrm{H}\right]$ menthol binding for two other TRPM8 mutants that were recently identified in a high-throughput random mutagenesis screen for residues specifically required for menthol activation ${ }^{29}$. A mutation in the C-terminal TRP domain (L1009R) that affects menthol efficacy rather than affinity ${ }^{29}$ resulted in normal $\left[{ }^{3} \mathrm{H}\right]$ menthol binding (Supplementary Fig. 3). In contrast, no specific $\left[{ }^{3} \mathrm{H}\right]$ menthol displacement was observed in membranes expressing mutant $\mathrm{Y} 745 \mathrm{H}$ (Supplementary Fig. 3), which supports the notion that this mutation in $\mathrm{S} 2$ affects menthol binding ${ }^{29}$.

From these data we can exclude the possibility that menthol acts through a nonspecific effect on plasma membrane lipids. Instead, expression of TRPM8 leads to specific $\left[{ }^{3} \mathrm{H}\right]$ menthol binding in plasma membrane-enriched fractions, and mutations that alter the menthol sensitivity of channel activation also affect the affinity of $\left[{ }^{3} \mathrm{H}\right]$ menthol binding.

\section{TRPM8-TRPM5 chimeras}

Notably, the three closest homologs of the cold- and mentholactivated TRPM8 (TRPM2, TRPM4 and TRPM5) are heatactivated $^{7,30}$ and insensitive to menthol (data not shown and ref. 29). Yet all basic residues in S4 and the S4-S5 linker are conserved among TRPM8, TRPM2, TRPM4 and TRPM5, including the residues involved in menthol activation (Arg842 and Lys856). Likewise, Tyr745 in S2 is fully conserved in all TRPM subfamily members. These observations imply that additional structural elements must underlie

the difference in menthol sensitivity among these channels. To address this question, we constructed chimerical channels in which specific regions in TRPM8 are substituted by the corresponding region of TRPM5. Transfer of larger regions of TRPM5 to TRPM8, such as the $\mathrm{C}$ terminus cytosolic tail, transmembrane segments $\mathrm{S} 1-\mathrm{S} 4$, or the pore region (S5 and S6), invariably led to nonfunctional channels, even in chimeras that apparently reached the plasma membrane (Supplementary Fig. 4 online). We therefore narrowed down our chimeric approach to the region encompassing S4 and the S4-S5 linker, and we transferred three small segments with relatively low sequence conservation from TRPM5 to TRPM8 (Fig. 5a). Only one chimeric protein (termed S4b_M5; Fig. 5a) expressed as a functional, depolarization-activated channel (Fig. $\mathbf{5 b}, \mathbf{c})$. At $25^{\circ} \mathrm{C}$, the activation curve of S4b_M5 was shifted toward more positive potentials compared with wild-type TRPM8 ( $V_{1 / 2}=226 \pm 12 \mathrm{mV} ; P<0.01$; Fig. 5c), but gating charge estimates $\left(z_{\mathrm{app}}=0.91 \pm 0.08 ; z_{\mathrm{T}}=0.93 \pm 0.07 ; n=6\right)$ were not significantly different $(P>0.2)$. Mutant S4b_M5 was activated upon cooling (Fig. 5d,e), and $V_{1 / 2}$ increased upon heating with a $\mathrm{d} V_{1 / 2} / \mathrm{d} T$ value of $6.1 \pm 0.7 \mathrm{mV}^{\circ} \mathrm{C}^{-1}$, which corresponds to a $\Delta S=-558 \pm 102$ $\mathrm{J} \mathrm{mol}^{-1} \mathrm{~K}^{-1}(n=5)$. Thus, in terms of temperature dependence, the S4b_M5 chimera retains the properties of TRPM8. However, mutant S4b_M5 showed a notable decrease in menthol sensitivity (Fig. 5f) and specific $\left[{ }^{3} \mathrm{H}\right]$ menthol binding (Supplementary Fig. 3). Fitting a Hill function to the dose dependence of the menthol-induced shift in $V_{1 / 2}$ (Fig. 5g) yielded a significantly higher $\mathrm{EC}_{50}$ value of $477 \pm 95 \mu \mathrm{M}$ and a significantly lower maximal shift of $84 \pm 8 \mathrm{mV}(n=6 ; P<0.01$ for both parameters).

\section{A TRPM8 gating model}

Mutation-induced changes in the $\mathrm{EC}_{50}$ for an agonist or even in the $\mathrm{IC}_{50}$ obtained from agonist displacement studies do not necessarily imply that the mutation affects the agonist binding site itself. Indeed, mutations that leave the affinity of the binding site unaffected but impair conformational changes downstream of agonist binding (that is, mutations that impair agonist efficacy) can lead to large increases in the $\mathrm{IC}_{50}$ and $\mathrm{EC}_{50}$ (ref. 31). To distinguish between these possibilities and obtain better insight into the effects of menthol on wild-type and mutant channels, we set out to develop a model that describes the combined effects of temperature, voltage and ligand binding on TRPM8 gating. We explored the predictions of both a HodgkinHuxley (HH)-type model and a Monod-Wyman-Changeux (MWC)type model ${ }^{32}$. For both models we assumed that each subunit of the tetrameric channel can independently bind a single menthol molecule. For simplicity, we made no distinction between the binding of menthol molecules to adjacent or diagonally opposed subunits. In the $\mathrm{HH}$ model, each of the four channel subunits independently 
a

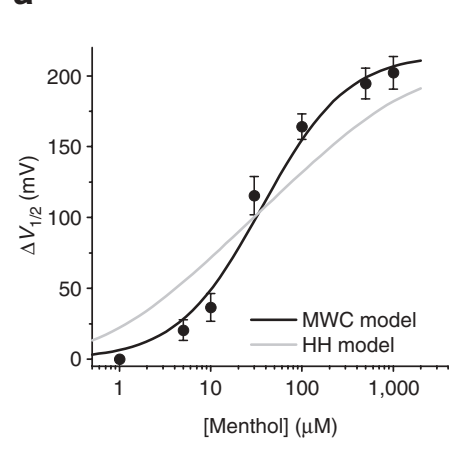

d

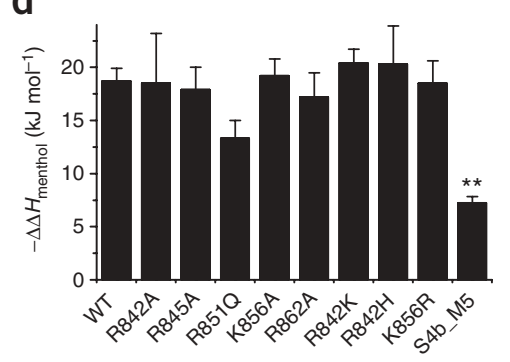

b

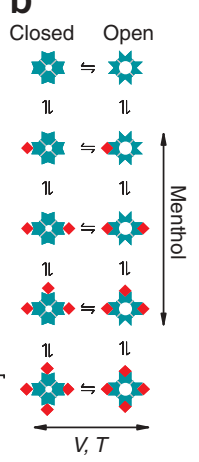

C

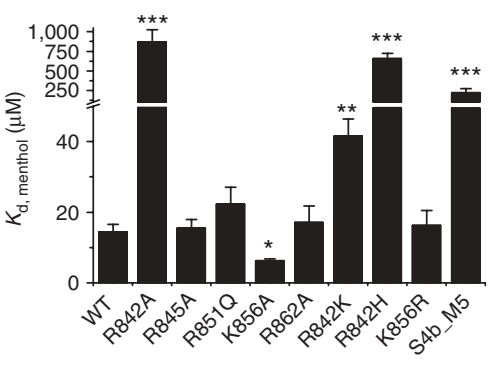

e

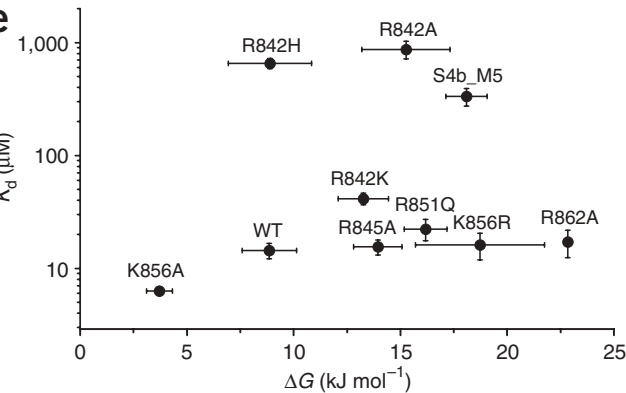

Figure 6 A quantitative model describing the combined effects of temperature, voltage and menthol on TRPM8. (a) Comparison of the best fit of an MWC model and an $\mathrm{HH}$ model to the wild-type data from Figure 4c. (b) Graphical representation of the MWC model. Shown is the homotetrameric TRPM8 channel in the closed and open configuration. Each of the four subunits can independently bind a single menthol molecule (red diamonds). For more details see text and Supplementary Methods.

(c,d) Parameters for the MWC model describing menthol affinity (c) and efficacy (d) were obtained by fitting the model to the data for wild-type and mutant TRPM8. The actual fits are shown together with the experimental data in Figures

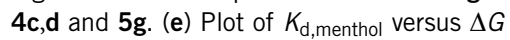
obtained in the absence of menthol at $25^{\circ} \mathrm{C}$. Error bars indicate s.e.m. $\left(n>5\right.$; ${ }^{*} P<0.05$; $\left.{ }^{* *} P<0.01 ;{ }^{* * *} P<0.001\right)$.

R845A, R851Q, R862A and K856R, we obtained values for $K_{\mathrm{d} \text {,menthol }}$ and $\Delta \Delta H_{\text {menthol }}$ that were not significantly different from those for wild-type TRPM8 (Fig. 6c,d), which indicates that neither menthol affinity nor menthol efficacy is affected by these mutations. Finally, in the case of the chimeric

undergoes a voltage-dependent activation reaction, and binding of menthol to a subunit shifts the voltage dependence of the activation reaction of that subunit toward lower voltages. For the channel to open, all four subunits have to be simultaneously in the activated state. In contrast, in the MWC model the four subunits undergo a concerted voltage-dependent transition between the closed and open states. In this model, stepwise binding of four menthol molecules leads to stepwise shifts of the voltage dependence of the concerted activation reaction toward lower voltages. A detailed formal description of both models can be found in Supplementary Methods.

Comparing the predictions of the $\mathrm{HH}$ and MWC models to the wild-type $\Delta V_{1 / 2}$ versus menthol data revealed that the MWC model provides a clearly superior fit to the experimental data (Fig. 6a,b). In comparison to the two-state model, two additional parameters must be included to explain the effects of ligand binding on steady state currents: (i) the menthol affinity of a single subunit in an open channel, which is determined by the affinity constant $K_{\mathrm{d} \text {,menthol, and }}$ (ii) the effect of menthol binding on channel gating, which is

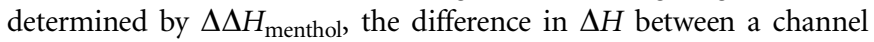
with menthol bound to each of the four subunits and a menthol-free channel. Thus, mutations that affect the menthol binding site should

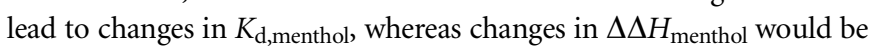
evidence for an altered menthol efficacy ${ }^{31}$. For wild-type TRPM8, the

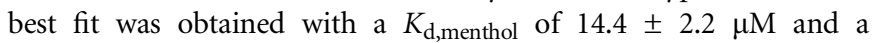
$\Delta \Delta H_{\text {menthol }}$ of $-18.7 \pm 1.2 \mathrm{~kJ} \mathrm{~mol}^{-1}$. Importantly, for mutants R842A, R842K and R842H we obtained significantly higher $K_{\mathrm{d} \text {,menthol }}$

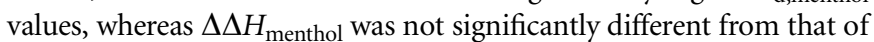
wild-type TRPM8 (Fig. 6c,d). These results strongly indicate that mutating the arginine side chain at position 842 leads to a significant decrease in the affinity of the menthol binding site, without changing the menthol efficacy. In contrast, we obtained a significantly $(P<0.05)$ reduced $K_{\mathrm{d} \text {,menthol }}$ for mutant K856A, again with an unchanged $\Delta \Delta H_{\text {menthol }}$ (Fig. 6c,d), which indicates that this mutation increases the affinity of the menthol binding site, again without effect on the efficacy of menthol binding on channel gating. For mutants channel S4b_M5, both $K_{\mathrm{d} \text {,menthol }}(220 \pm 51 \mu \mathrm{M})$ and $\Delta \Delta H_{\text {menthol }}$ $\left(-7.2 \pm 0.7 \mathrm{~kJ} \mathrm{~mol}^{-1}\right)$ were significantly different from those of wildtype TRPM8 $(P<0.01$ and $P<0.001$, respectively; Fig. $6 \mathbf{c}$,d), which indicates that residues in this segment determine both menthol affinity and efficacy.

Importantly, there was no obvious correlation between $K_{\mathrm{d} \text {,menthol }}$ and the $\Delta G$ values obtained in menthol-free conditions (Fig. 6e; correlation coefficient $r=0.102$ ), which indicates that the observed changes in menthol affinity are not a mere consequence of alterations in the relative stability of the closed and open states. Thus, based on our functional data and modeling we conclude that mutations at Arg842 and Lys856 specifically affect the affinity of the TRPM8 menthol binding site.

The MWC model also faithfully reproduces other known effects of menthol on TRPM8 gating $8,14,15$, including a shift of the cold sensitivity of inward currents toward higher temperatures, a parallel leftward shift of the activation curves and a parallel downward shift of the $V_{1 / 2}$ versus $T$ curves (see Supplementary Fig. 5 online). Moreover, the voltage-dependent MWC model can also describe ligand activation of other voltage-dependent thermoTRPs, including the effects of capsaicin on TRPV1 (see Supplementary Fig. 5). In the absence of ligands, the voltage-dependent MWC model becomes equivalent to the two-state channel-gating model that we have previously shown to accurately predict the temperature-dependent gating of TRPM8 and other thermoTRPs ${ }^{7,8}$.

\section{DISCUSSION}

Mammals detect changes in environmental temperature through sensory nerve endings in the skin and mouth ${ }^{1}$ and through skin keratinocytes $^{2}$, and thermoTRPs have been identified as the main temperature sensors in these cells $s^{1-4,33,34}$. A notable feature of thermoTRPs is that they react not only to changes in temperature but also to a variety of natural and synthetic chemicals. This property is believed to underlie different forms of chemesthesis, such as the hotness of capsaicin (the pungent compound in chili peppers, which 
activates TRPV1) or the freshness of menthol. Recent studies indicate that most thermoTRPs are voltage-sensitive, and that chemical and thermal stimuli act on these channels by altering the voltage dependence of activation ${ }^{6-10}$. However, at the onset of this study, the mechanisms and structures underlying voltage sensing in TRP channels were fully unknown.

So how can thermoTRPs integrate thermal, chemical and voltage stimuli? Our present study provides important new mechanistic insight, using the cold- and menthol-sensitive TRPM8 as a paradigm. First, we identified residues in S4 and the S4-S5 linker that significantly contribute to the gating charge. These data provide the first functional evidence that this region forms part of the voltage sensor in TRP channels. Second, we demonstrated that mutations that alter voltage sensitivity and gating charge also affect the channel's sensitivity to temperature. These results confirm and expand the previous hypothesis that voltage and temperature act on the same gating step $^{8}$. Third, we showed that specific mutations in S4 and the S4-S5 linker strongly affect the channel's sensitivity and affinity for menthol. This finding suggests that menthol acts on TRPM8 through a direct interaction with the voltage sensor. Finally, we have presented a quantitative model that accurately describes the combined actions of temperature, voltage and menthol on TRPM8 gating. We believe this model will be highly instrumental for the interpretation of further structure-function work on thermoTRPs and for modeling the electrical behavior of thermosensitive neurons.

The finding that positively charged residues in S4 and the S4-S5 linker of TRPM8 are involved in voltage sensing may not have been fully unexpected, considering the homology between this segment and the voltage sensor of $\mathrm{Kv}$ channels (Fig. 1a,b), and it is therefore tempting to speculate that voltage-dependent gating in Kv and TRP channels occurs following a similar principle. Yet our data also point out some significant differences between these two types of voltagegated channels. First, the total gating charge of TRPM8 $\left(0.89 e_{\mathrm{o}}\right)$ is one order of magnitude lower than what has been determined for classical voltage-gated $\mathrm{K}^{+}, \mathrm{Ca}^{2+}$ and $\mathrm{Na}^{+}$channels $\left(7-14 e_{\mathrm{o}}\right)^{19,20,35-38}$. The total gating charge of other voltage-sensitive TRP channels has not yet been scrutinized using LSM or similar techniques, but available data suggest that all of them have gating charges below $1 e_{\mathrm{o}}$ (refs. 7-9,39). Second, neutralizing Arg842 or Lys856 led to a reduction of the gating charge of only $0.2-0.3 e_{\mathrm{o}}$. Assuming that all four subunits of a homotetrameric TRPM8 channel contribute equally to the gating charge, it follows that these voltage-sensing residues travel across less than $8 \%$ of the transmembrane electrical field. This contrasts sharply with $\mathrm{Kv}$ channels, in which the four voltage-sensing arginines in S4 almost entirely cross the transmembrane electrical field ${ }^{18,21}$. Although the absolute reduction in gating charge upon charge neutralization may seem small, the relative reduction amounts to $\sim 30 \%$, which is similar to what was observed upon neutralizing basic residues in S4 of Shakertype $\mathrm{K}^{+}$and other voltage-gated channels. Finally, we measured the largest reduction in gating charge upon neutralizing Lys856, which on the basis of sequence homology is expected to be located in the S4-S5 linker rather than in the S4 segment. This may indicate that in TRPM8 the S4-S5 linker has a more central role in voltage sensing than in Kv channels, in which the S4 segment is believed to carry the entire gating charge. Alternatively, the tertiary structure of the voltage sensor region may not be fully conserved between $\mathrm{Kv}$ and TRP channels. For example, S4 of TRPM8 may extend further to the C terminus such that it includes Lys856.

An important finding of our present study is that mutations in S4 and the S4-S5 linker of TRPM8 can specifically affect the affinity of the menthol binding site. Using a high-throughput random mutagenesis screen, investigators ${ }^{29}$ recently identified Tyr745 in S2 as an important determinant of menthol affinity. Notably, in the crystal structure of Kv1.2 the S2 and S4 domains are in close proximity, and the side chain of the third S4 arginine (Arg300), which corresponds to Arg842 of TRPM8, points toward S2 (refs. 22,23). These findings raise the possibility that a single menthol molecule intercalates between $\mathrm{S} 2$ and S4, interacting simultaneously with residues on both transmembrane segments, although allosteric effects on a distal menthol binding site cannot be fully excluded. Clearly, structural data is required to validate the location of the menthol binding site on TRPM8.

Mutations in the transmembrane segments (S1-S4) not only affect activation of TRPM8 by menthol and icilin ${ }^{29,40}$ but also affect activation of the heat-activated TRPV1 by capsaicin and related vanilloids $^{5,41}$. Moreover, a recent study used chimeras between TRPV1 and TRPM8 to demonstrate that the C termini of these channels are not crucial for activation by capsaicin and menthol, respectively ${ }^{42}$. These findings suggest a more general mechanism, in which TRP channel ligands shift the voltage dependence of channel activation through binding to the $\mathrm{S} 1-\mathrm{S} 4$ region. In the distally related hyperpolarization-activated cyclic nucleotide-gated (HCN) channels and large conductance $\mathrm{Ca}^{2+}$-activated $(\mathrm{BK}) \mathrm{K}^{+}$channels, ligand binding also leads to a shift in the voltage dependence of channel activation. However, in these channels, the ligands act through binding to well-defined C-terminal intracellular domains, which are clearly separated from the S4 voltage sensor ${ }^{43,44}$. This fundamental difference in the mechanism of ligand-dependent gating probably reflects the fundamental difference in the physicochemical properties of the activating ligands: $\mathrm{Ca}^{2+}$ and cyclic nucleotides are hydrophilic, membrane-impermeant intracellular messengers, whereas exogenous TRP channel ligands such as menthol and capsaicin are hydrophobic compounds that easily enter and permeate biological membranes.

All tested charge-neutralizing mutations in TRPM8 altered the voltage dependence and thermal sensitivity, including those that did not affect gating charge or menthol affinity. For example, neutralization of Arg851 and Arg862 strongly shifted $V_{1 / 2}$ to more positive potentials and changed the apparent thermal thresholds to significantly lower temperatures, which is indicative of a strong increase in the difference in free energy $(\Delta G)$ between the open and closed states (Supplementary Table 1). One possible explanation is that Arg851 and Arg862 stabilize the open state via the formation of salt bridges with one or several of the 19 acidic residues that are present in the transmembrane region of TRPM8. Evidence for such electrostatic interactions between positive charges in S4 and acidic residues in S2 and S3 has been previously presented for the Shaker-type $\mathrm{K}^{+}$channel $^{45}$. Accordingly, the unaltered $\Delta G$ values for charge-conserving mutations at these positions (R851K and R862K; Supplementary Table 1) could be explained by the fact that lysines can substitute for arginines in the formation of salt bridges. Alternatively, neutralization of the positive residues may allosterically affect the stability of the open and/or closed state.

Voltage-gated channels involved in rapid cellular processes such as generation and conduction of action potentials, muscle contraction or neurotransmitter release act almost like a switch that turns on and off in response to small voltage excursions ${ }^{27}$. Such steep voltage dependence requires a high gating charge ${ }^{18,27}$. In contrast, TRPM8 and other thermoTRPs carry a much lower gating charge and thus have much shallower voltage dependence ${ }^{7-9,24}$. A thermodynamic consequence of a low gating charge is that changes in $\Delta G$ (for example, as a result of ligand binding, mutagenesis or variations in temperature) result in large shifts in the voltage dependence (equation (2)). In the case of thermoTRPs, in which $\Delta S$ is substantial $\left(-560 \mathrm{~J} \mathrm{~mol}^{-1} \mathrm{~K}^{-1}\right.$ for TRPM8, 
and between +400 and $+600 \mathrm{~J} \mathrm{~mol}^{-1} \mathrm{~K}^{-1}$ for the heat-activated TRPV1, TRPM4 and TRPM5) ${ }^{7}$, this translates into a steep temperature dependence of inward currents at physiological voltages. The structures and mechanisms that determine whether a thermoTRP is cold-activated $(\Delta S<0)$ or heat-activated $(\Delta S>0)$ are currently elusive, but, notably, a recent study suggests that they may be contained in the C-terminal tail of the channel ${ }^{42}$. Other notable examples of ion channels with low gating charges are the recently cloned voltage-gated proton channels $\left(\mathrm{H}_{\mathrm{v}} 1\right.$ and $\left.\mathrm{mVSOP}\right)$, which are four transmembrane proteins homologous to the voltage sensor domain (S1-S4) of voltage-gated channels ${ }^{46,47}$. The reported apparent gating charge of $\mathrm{H}_{\mathrm{v}} 1$ is similar to that of TRPM8 $(\sim 0.9$ equivalent charges), and a notable property of this channel is its strong temperature dependence ${ }^{47}$. We propose that variations in the gating charge carried by a conserved voltage sensor region optimize structurally related cation channels for sensing either voltage or temperature.

\section{METHODS}

Channel constructs, electrophysiology and intracellular $\mathrm{Ca}^{2+}$ measurements. HEK 293 cells were grown in DMEM containing 10\% (v/v) fetal calf serum, $4 \mathrm{mM}$ L-alanyl-L-glutamine, 100 units $\mathrm{ml}^{-1}$ penicillin and $100 \mu \mathrm{g} \mathrm{ml}^{-1}$ streptomycin at $37{ }^{\circ} \mathrm{C}$ in a humidity-controlled incubator with $10 \% \mathrm{CO}_{2}$. Cells were transiently transfected with human TRPM8 cloned in the bicistronic pCAGGS-IRES-GFP vector using TransIT-293 transfection reagent (Mirus). Single amino acids in TRPM8 were mutated using the standard PCR overlap extension technique ${ }^{48}$, and the nucleotide sequences of all mutants were verified by DNA sequencing.

Between $16 \mathrm{~h}$ and $24 \mathrm{~h}$ after transfection, currents were recorded in the whole-cell configuration of the patch-clamp technique using an EPC-9 amplifier and PULSE software (HEKA Elektronik). Data were sampled at $5-20 \mathrm{kHz}$ and digitally filtered off-line at $1-5 \mathrm{kHz}$. Between 70 and $90 \%$ of the series resistance was compensated, thereby reducing voltage errors to less than $10 \mathrm{mV}$. All patch-clamp experiments were performed using identical intra- and extracellular solutions containing (in $\mathrm{mM}$ ) $150 \mathrm{NaCl}, 5 \mathrm{MgCl}_{2}, 5$ EGTA and 10 HEPES, pH 7.4. Under these ionic conditions, open TRPM8 channels show a linear current-voltage relation that reverses at $0 \mathrm{mV}$. The temperature of the perfusate was controlled using an SC-20 dual in-line heater/cooler (Warner Instruments). Reported temperature was measured using a TA-29 thermistor (Thermometrics) placed within $500 \mu \mathrm{m}$ of the patch-clamped cell.

The extracellular solution used in ratiometric $\left[\mathrm{Ca}^{2+}\right]_{i}$ measurements contained (in $\mathrm{mM}$ ) $150 \mathrm{NaCl}, 6 \mathrm{KCl}, 2 \mathrm{CaCl}_{2}, 1.5 \mathrm{MgCl}_{2}, 10$ glucose and 10 HEPES, pH 7.4. Cells were incubated with $2 \mu \mathrm{M}$ Fura-2 acetoxymethyl ester for $30 \mathrm{~min}$ at $37^{\circ} \mathrm{C}$. The fluorescent signal of a single cell was measured upon alternating illumination at 350 and $380 \mathrm{~nm}$ using a Polychrome IV monochromator and photodiode (TILL Photonics). Calculations of $\left[\mathrm{Ca}^{2+}\right]_{\mathrm{i}}$, including the correction for the temperature-dependent changes in Fura-2 affinity, were performed as previously described ${ }^{49}$.

Radioactive menthol binding assay. Radioligand binding assays were performed as described previously with small modification $s^{28}$. Briefly, transfected HEK 293 cells were collected by centrifugation at $500 \mathrm{~g}$ for $5 \mathrm{~min}$ at $4{ }^{\circ} \mathrm{C}$ (all subsequent steps were carried out at $4{ }^{\circ} \mathrm{C}$ ) and resuspended in $3 \mathrm{ml}$ ice-cold lysis buffer, which contained (in $\mathrm{mM}$ ) $10 \mathrm{KCl}, 1.5 \mathrm{MgCl}_{2}, 1$ EDTA, 1 phenylmethylsulfonyl fluoride, 50 Tris $(\mathrm{pH} 7.5)$ and a protease inhibitor cocktail $\left(10 \mu \mathrm{g} \mathrm{ml}^{-1}\right.$ leupeptin and antipain, $2 \mu \mathrm{g} \mathrm{ml}^{-1}$ chymostatin and pepstatin). After rotation on an end-over-end stirrer for $30 \mathrm{~min}$, cells were subjected to three freeze-thaw cycles and then passed ten times through a 26-gauge needle. The resulting lysates were centrifuged at $4,000 \mathrm{~g}$ for $15 \mathrm{~min}$ to remove nuclei, mitochondria and any remaining large cellular fragments. Obtained supernatants were ultracentrifuged at $20,000 \mathrm{~g}$ for $20 \mathrm{~min}$, yielding a low-speed pellet membrane fraction that was solubilized in cold phosphate-buffered saline,

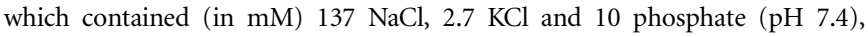
supplemented with $1 \%$ Triton X-100, $0.2 \%$ sodium dodecyl sulfate, $1 \mathrm{mM}$ PMSF and the protease inhibitor cocktail. In western blot experiments we confirmed that this low-speed pellet was a plasma membrane-enriched fraction that contained more than 95\% of expressed TRPM8 and endogenous PMCA1, a plasma membrane $\mathrm{Ca}^{2+}$-transporting ATPase (data not shown). Low-speed pellet $(2 \mu \mathrm{g})$ was incubated for $30 \mathrm{~s}$ at room temperature $\left(22-24^{\circ} \mathrm{C}\right)$ in $500 \mu \mathrm{l}$ of $50 \mathrm{mM}$ Tris- $\mathrm{HCl}(\mathrm{pH} 7.5)$ buffer supplemented with $25 \mathrm{nM}\left[{ }^{3} \mathrm{H}\right]$ menthol (ViTrax) in the absence or presence of nonradioactive menthol over a concentration range of $10 \mu \mathrm{M}$ to $5 \mathrm{mM}$. In control experiments we found that a 30-s incubation period is amply sufficient to reach maximal specific menthol binding, in accordance with patch-clamp experiments showing that the binding/unbinding of menthol to TRPM8 is very fast (time constants < $2 \mathrm{~s}$ ). Total radioactivity measured as disintegrations per minute added to each assay was around 100,000 . Incubations were terminated by filtration through nitrocellulose membrane filters (Millipore) presoaked with $150 \mathrm{mM} \mathrm{KCl}$ by use of the 1225 sampling manifold (Millipore) and washed three times with icecold $50 \mathrm{mM}$ Tris- $\mathrm{HCl}$ ( $\mathrm{pH}$ 7.5) buffer. The filters were placed individually into vials containing $3 \mathrm{ml}$ EcoLite scintillation liquid (ICN Biomedicals), and the amount of radioactivity bound to the filters was quantified by liquid scintillation spectrophotometry on a Tri Carb 2900 TR liquid scintillation analyzer (Packard Biosciences).

Data analysis. Data analysis was performed using Origin 7.0 (OriginLab Corporation). Group data are expressed as mean \pm s.e.m. from $n$ independent experiments. The Student's unpaired, two-tailed $t$-test was used for statistical comparison, and $P<0.05$ was considered statistically significant. $P_{\text {open }}$ was determined from steady state current-voltage relations as $G / G_{\max }$, where $G_{\max }$ represents the maximal steady state conductance, which was obtained at strongly depolarized potentials (between +160 and $+260 \mathrm{mV}$ ) in the presence of $1 \mathrm{mM}$ menthol. We refrained from using tail currents to determine TRPM8 activation curves, as we found that very rapid tail current deactivation, especially at temperatures $>20{ }^{\circ} \mathrm{C}$ ( ref. 8), can lead to serious underestimation of $P_{\text {open }}$ (Supplementary Fig. 1).

Note: Supplementary information and chemical compound information is available on the Nature Chemical Biology website.

\section{ACKNOWLEDGMENTS}

We thank G. Droogmans, F. Mahieu, J. Vriens, R. Vennekens and J. Prenen for helpful suggestions and criticisms, and A. Patapoutian and M. Bandell for the Y745H and L1009R mutants. This work was supported by grants from the Human Frontiers Science Program (RGP32/2004), the Belgian Federal Government (IUAP P5/05), the Research Foundation-Flanders (G.0136.00, G.0172.03 and G.0565.07) and the Research Council of the KU Leuven (GOA2004/07 and EF/95/010).

\section{AUTHOR CONTRIBUTIONS}

T.V. designed, performed and analyzed the electrophysiological experiments. G.O. developed the radioligand binding assay and conducted the biochemical experiments and molecular modeling. A.J. designed and performed TRPM8 mutagenesis. T.V. and K.T. performed the mathematical modeling. T.V. wrote the manuscript; G.O., K.T. and B.N. edited the manuscript; and T.V. and B.N. supervised the project.

\section{COMPETING INTERESTS STATEMENT}

The authors declare that they have no competing financial interests.

Published online at http://www.nature.com/naturechemicalbiology

Reprints and permissions information is available online at http://npg.nature.com/ reprintsandpermissions

1. Julius, D. \& Basbaum, A.I. Molecular mechanisms of nociception. Nature 413, 203-210 (2001).

2. Moqrich, A. et al. Impaired thermosensation in mice lacking TRPV3, a heat and camphor sensor in the skin. Science 307, 1468-1472 (2005).

3. Clapham, D.E. TRP channels as cellular sensors. Nature 426, 517-524 (2003).

4. Patapoutian, A., Peier, A.M., Story, G.M. \& Viswanath, V. ThermoTRP channels and beyond: mechanisms of temperature sensation. Nat. Rev. Neurosci. 4, 529-539 (2003).

5. Jordt, S.E., McKemy, D.D. \& Julius, D. Lessons from peppers and peppermint: the molecular logic of thermosensation. Curr. Opin. Neurobiol. 13, 487-492 (2003).

6. Voets, T., Talavera, K., Owsianik, G. \& Nilius, B. Sensing with TRP channels. Nat. Chem. Biol. 1, 85-92 (2005)

7. Talavera, K. et al. Heat activation of TRPM5 underlies thermal sensitivity of sweet taste. Nature 438, 1022-1025 (2005). 
8. Voets, T. et al. The principle of temperature-dependent gating in cold- and heatsensitive TRP channels. Nature 430, 748-754 (2004).

9. Chung, M.K., Guler, A.D. \& Caterina, M.J. Biphasic currents evoked by chemical or thermal activation of the heat-gated ion channel, TRPV3. J. Biol. Chem. 280, 15928-15941 (2005).

10. Nilius, B. et al. The $\mathrm{Ca}^{2+}$-activated cation channel TRPM4 is regulated by phosphatidylinositol 4,5-biphosphate. EMBO J. 25, 467-478 (2006).

11. Xu, H., Blair, N.T. \& Clapham, D.E. Camphor activates and strongly desensitizes the transient receptor potential vanilloid subtype 1 channel in a vanilloid-independent mechanism. J. Neurosci. 25, 8924-8937 (2005).

12. Rohacs, T., Lopes, C.M., Michailidis, I. \& Logothetis, D.E. PI $(4,5) \mathrm{P}_{2}$ regulates the activation and desensitization of TRPM8 channels through the TRP domain. Nat. Neurosci. 8, 626-634 (2005).

13. Nilius, B. et al. Gating of TRP channels: a voltage connection? J. Physiol. (Lond.) 567, 35-44 (2005).

14. Peier, A.M. et al. A TRP channel that senses cold stimuli and menthol. Cell 108, 705-715 (2002)

15. McKemy, D.D., Neuhäusser, W.M. \& Julius, D. Identification of a cold receptor reveals a general role for TRP channels in thermosensation. Nature 416, 52-58 (2002).

16. Montell, C. The TRP superfamily of cation channels. Sci. STKE 2005, re3 (2005).

17. Yellen, G. The voltage-gated potassium channels and their relatives. Nature 419 , 35-42 (2002).

18. Bezanilla, F. Voltage-gated ion channels. IEEE Trans. Nanobioscience 4, 34-48 (2005).

19. Aggarwal, S.K. \& MacKinnon, R. Contribution of the S4 segment to gating charge in the Shaker K+ channel. Neuron 16, 1169-1177 (1996).

20. Seoh, S.A., Sigg, D., Papazian, D.M. \& Bezanilla, F. Voltage-sensing residues in the S2 and S4 segments of the Shaker K+ channel. Neuron 16, 1159-1167 (1996).

21. MacKinnon, R. Structural biology. Voltage sensor meets lipid membrane. Science $\mathbf{3 0 6}$, 1304-1305 (2004)

22. Long, S.B., Campbell, E.B. \& MacKinnon, R. Voltage sensor of Kv1.2: structural basis of electromechanical coupling. Science 309, 903-908 (2005).

23. Long, S.B., Campbell, E.B. \& MacKinnon, R. Crystal structure of a mammalian voltagedependent Shaker family K+ channel. Science 309, 897-903 (2005).

24. Brauchi, S., Orio, P. \& Latorre, R. Clues to understanding cold sensation: thermodynamics and electrophysiological analysis of the cold receptor TRPM8. Proc. Natl. Acad. Sci. USA 101, 15494-15499 (2004).

25. Correa, A.M., Bezanilla, F. \& Latorre, R. Gating kinetics of batrachotoxin-modified $\mathrm{Na}^{+}$ channels in the squid giant axon. Voltage and temperature effects. Biophys. J. 61, 1332-1352 (1992).

26. Almers, W. Gating currents and charge movements in excitable membranes. Rev. Physiol. Biochem. Pharmacol. 82, 96-190 (1978).

27. Sigworth, F.J. Voltage gating of ion channels. Q. Rev. Biophys. 27, 1-40 (1994).

28. Wright, C.E., Bowen, W.P., Grattan, T.J. \& Morice, A.H. Identification of the L-menthol binding site in guinea-pig lung membranes. Br. J. Pharmacol. 123, 481-486 (1998).

29. Bandell, M. et al. High-throughput random mutagenesis screen reveals TRPM 8 residues specifically required for activation by menthol. Nat. Neurosci. 9, 493-500 (2006).
30. Togashi, K. et al. TRPM2 activation by cyclic ADP-ribose at body temperature is involved in insulin secretion. EMBO J. 25, 1804-1815 (2006).

31. Colquhoun, D. Binding, gating, affinity and efficacy: the interpretation of structureactivity relationships for agonists and of the effects of mutating receptors. Br. J. Pharmacol. 125, 924-947 (1998).

32. Hille, B. Ion Channels of Excitable Membranes (Sinauer Associates, Sunderland, Massachusetts, USA, 2001).

33. Lee, H., lida, T., Mizuno, A., Suzuki, M. \& Caterina, M.J. Altered thermal selection behavior in mice lacking transient receptor potential vanilloid 4. J. Neurosci. 25 1304-1310 (2005)

34. Caterina, M.J. et al. Impaired nociception and pain sensation in mice lacking the capsaicin receptor. Science 288, 306-313 (2000).

35. Schoppa, N.E., McCormack, K., Tanouye, M.A. \& Sigworth, F.J. The size of gating charge in wild-type and mutant Shaker potassium channels. Science 255, 1712-1715 (1992).

36. Islas, L.D. \& Sigworth, F.J. Voltage sensitivity and gating charge in Shaker and Shab family potassium channels. J. Gen. Physiol. 114, 723-742 (1999).

37. Hirschberg, B., Rovner, A., Lieberman, M. \& Patlak, J. Transfer of twelve charges is needed to open skeletal muscle $\mathrm{Na}^{+}$channels. J. Gen. Physiol. 106, 1053-1068 (1995).

38. Noceti, F. et al. Effective gating charges per channel in voltage-dependent $\mathrm{K}^{+}$and $\mathrm{Ca}^{2+}$ channels. J. Gen. Physiol. 108, 143-155 (1996).

39. Nilius, B. et al. Voltage dependence of the $\mathrm{Ca}^{2+}$-activated cation channel TRPM4. J. Biol. Chem. 278, 30813-30820 (2003).

40. Chuang, H.H., Neuhausser, W.M. \& Julius, D. The super-cooling agent icilin reveals a mechanism of coincidence detection by a temperature-sensitive TRP channel. Neuron 43, 859-869 (2004).

41. Gavva, N.R. et al. Molecular determinants of vanilloid sensitivity in TRPV1. J. Biol. Chem. 279, 20283-20295 (2004).

42. Brauchi, S., Orta, G., Salazar, M., Rosenmann, E. \& Latorre, R. A hot-sensing cold receptor: C-terminal domain determines thermosensation in transient receptor potential channels. J. Neurosci. 26, 4835-4840 (2006).

43. Rothberg, B.S. Allosteric modulation of ion channels: the case of maxi-K. Sci. STKE 2004, pe16 (2004).

44. Robinson, R.B. \& Siegelbaum, S.A. Hyperpolarization-activated cation currents: from molecules to physiological function. Annu. Rev. Physiol. 65, 453-480 (2003).

45. Papazian, D.M. et al. Electrostatic interactions of S4 voltage sensor in Shaker $\mathrm{K}^{+}$ channel. Neuron 14, 1293-1301 (1995).

46. Sasaki, M., Takagi, M. \& Okamura, Y. A voltage sensor-domain protein is a voltagegated proton channel. Science 312, 589-592 (2006).

47. Ramsey, I.S., Moran, M.M., Chong, J.A. \& Clapham, D.E. A voltage-gated protonselective channel lacking the pore domain. Nature 440, 1213-1216 (2006).

48. Ho, S.N., Hunt, H.D., Horton, R.M., Pullen, J.K. \& Pease, L.R. Site-directed mutagenesis by overlap extension using the polymerase chain reaction. Gene 77, 51-59 (1989).

49. Vriens, J. et al. Cell swelling, heat, and chemical agonists use distinct pathways for the activation of the cation channel TRPV4. Proc. Natl. Acad. Sci. USA 101, 396-401 (2004). 